\title{
Infant's psychophysiological profile and temperament at 3 and 12 months
}

\author{
Raquel Costa*, Bárbara Figueiredo ${ }^{1}$ \\ University of Minho, School of Psychology, Campus de Gualtar, 4710-057 Braga, Portugal
}

\section{A R T I C L E I N F O}

\section{Article history:}

Received 5 August 2009

Received in revised form 13 April 2010

Accepted 6 January 2011

\section{Keywords:}

Infant temperament

Mother-infant interaction

Neurobehavior

Neuroendocrine reactivity

Newborn

Social withdrawal

\begin{abstract}
A B S T R A C T
This study is intended to analyze (1) differences in infant temperament at 3 and 12 months according to infants' psychophysiological profiles: "withdrawn" "extroverted", and "underaroused" and (2) changes in infant temperament from 3 to 12 months, namely according to the infant psychophysiological profile and the quality of mother-infant interaction. Ninetyfour 8-week-old infants were assessed using the Neonatal Behavioral Assessment Scale (NBAS, Brazelton \& Nugent, 1995) and the Alarm Distress Baby Scale (ADBB, Guedeney \& Fermanian, 2001). Saliva samples were collected at 8-12 weeks old, both before and after a routine inoculation for cortisol reactivity measurement. Mother-infant interaction was evaluated at 12-16 weeks, using the Global Rating Scales (GRS, Murray, Fiori-Cowley, Hooper, \& Cooper, 1996). Mothers' reports on infant temperament at 3 and 12 months were collected using the Infant Behavior Questionnaire (IBQ, Rothbart, 1981). Significant differences in mothers' perception of infant temperament were found at both 3 and 12 months in infants with distinct psychophysiological profiles. Stability was observed in most of the temperament's dimensions from 3 to 12 months old; still, there were changes in mothers' perception of infant temperament in terms of level of distress, cuddliness, sadness and approach. Infant psychophysiological profile and mother-infant interaction both influence with the pattern of those changes. The results corroborate that infant's characteristics early in life as well as contextual factors influence with mothers' perception of infant temperament and with changes across the 1 st year of life.
\end{abstract}

(C) 2011 Elsevier Inc. All rights reserved.

There is no clear consensus about the nature of the temperament construct, although it is almost completely accepted that temperament has early development and biologic roots (Goldsmith et al., 1987), even if its characteristics may change over time. Several approaches to the construct have been advanced; Thomas, Chess, and Birch (1968) argue that infants begin to express themselves as individuals from the time of birth and show distinct individuality in temperament in the first weeks of life. Furthermore, they claim that at as early as the age of two or three months there is a discernible behavioral profile or temperament: "easy", "difficult", and "slow to warm up". The "easy" children are characterized by a positive mood, regularity, low/moderate intensity of reaction, adaptability and approach. The "difficult" children are irregular, have intense reactions, withdraw from stimuli, have troubles adapting and a negative mood. The "slow to warm up" show a low activity level, only withdraw at the first exposure to stimuli, are slow to adapt, have a mildly negative mood and low intensity reactions. These authors hypothesized that personality is shaped by the constant interplay of temperament and environment. If there is harmony between the infant's characteristics and the environment, the child is expected to show a healthy development. Behavioral disorders appear when there is a conflict between the child's temperament and the environmental demands.

\footnotetext{
* Corresponding author. Tel.: +351 253678987.

E-mail addresses: rcosta@psi.uminho.pt (R. Costa), bbfi@psi.uminho.pt (B. Figueiredo).

1 Tel.: +351253678987.
} 
Later on, in the 1980s, Rothbart's (1981) developed a psychobiological oriented approach to temperament that emphasizes primarily biological based individual differences in reactivity and self-regulation (Rothbart, Derryberry, \& Posner, 1994; Rothbart \& Posner, 1985). Behaviorally, temperament is relatively stable and can be observed as a set of individual differences in the emotionality, activity, and attention patterns (Goldsmith et al., 1987).

Buss and Plomin (1984), defined temperament as a set of inherent personality traits of genetic origin that appear during the first year of life. On the other hand, Goldsmith and Campos (1986) conceived temperament as emotional in nature, referring to individual differences in the probability of experiencing and expressing the primary emotions and arousal.

Almost completely agreement exists between these approaches in some aspects of temperament, namely that temperament refers to individual differences, is biologically based, is a component of personality and may change over time and that temperamental dimensions reflect behavioral tendencies. On the other hand, divergences are essentially related with the temperament dimensions and boundaries for temperament (Goldsmith et al., 1987).

Although these theories give us a direction on the nature of factors that might be involved in the development of temperament, there is still no evidence regarding exactly which are those factors. In this study we intend to contribute to further knowledge on this question by analyzing the influence of infant psychophysiology and environmental factors on temperament development over the 1st year of life.

\section{Infant characteristics and infant temperament}

Because temperament has been found to be related to a variety of difficulties such as attachment security (Seifer, Schiller, Sameroff, Resnick, \& Riordan, 1996), internalization/externalization behavior problems (Belsky, Hsieh, \& Crnic, 1998), hyperactivity (Wolke, Rizzo, \& Woods, 2002), depression (Gartstein \& Bateman, 2008) personality and social development difficulties (Kagan, 1998) and anxiety disorders (Biederman et al., 1993; Kagan, Snidman, Zentner, \& Peterson, 1999; Lindhout, Markus, Hoogendijk, \& Boer, 2009; Rosenbaum et al., 1993), the study of infant characteristics associated with infant temperament is of great interest.

Studies on infant characteristics rely almost exclusively on gender differences: boys exhibit higher activity and approach levels (Campbell \& Eaton, 1999; Maziade, Boudreault, Thivierge, Caperaa, \& Cote, 1984), whereas girls show higher hesitation when approaching novel objects (Martin, Wisenbaker, Baker, \& Huttunen, 1997; Rothbart, 1988). In a recent meta-analysis to estimate the magnitude of gender differences on temperament Else-Quest, Hyde, Goldsmith, and Van Hulle (2006) found that girls have higher inhibitory control and perceptual sensitivity while boys have higher activity and high intensity pleasure.

\subsection{Stability and change in individual differences}

Another major empirical issue in infant temperament research concerns the stability of individual differences (Bates, 1987). Some authors argue that the original characteristics of temperament tend to remain constant in quality over the years, although environmental circumstances may influence the infants' reactions and behavior throughout the development (Thomas et al., 1968). As a child develops, there are manifestations of temperamental change over time, with rapid development during infancy (Rothbart, 1989). Some dimensions of temperament such as positive emotionality, level of activity, approach, distress to limitations, and fear tend to increase throughout the first year of life (Carranza, Perez-Lopez, Gonzalez, \& Martinez-Fuentes, 2000; Rothbart, 1986, 1988, 1989), while others, like attention and orientation develop in a U-shape curve in the first year of life (Ruff \& Rothbart, 1996). Greater stability was found in aggregated scores, particularly in those reflecting difficultness (Lee \& Bates, 1985) or emotionality (Matheny, Riese, \& Wilson, 1985; Riese, 1987). The continuation of a child being classified as temperamentally difficult was found to be of about $50 \%$ at ages from 6 to 24 months (Lee \& Bates, 1985; McNeil \& Persson-Blennow, 1988).

(Dis)Continuity in infant temperament, and the comprehension of the variables that influence it, is an extremely relevant challenge within developmental theory and research. Some researchers attempted to answer the question of what are the conditions for continuity and discontinuity in infant temperament. Matheny (1986) found that infants' who had become less negative, more attentive, and more socially oriented at an age from 12 to 24 months, came from more emotionally cohesive families and their mothers were more expressive and involved with them. Similarly, Washington, Minde, and Goldberg (1986) reported that preterm babies who became less difficult over time (compared with premature infants that became more difficult) had more sensitive mothers. Engfer (1986) related that mothers who perceived their infants as becoming more difficult between 4 and 18 months were less sensitive and experienced more marital problems, while mothers perceiving their infants as becoming less difficult were more relaxed, optimistic and less irritable. Feldman, Greenbaum, Mayes, and Erlich (1997) found that a decrease on infant difficultness from 3 to 9 months was associated with increases on mother's sensitivity and decreases on maternal intrusiveness in the interaction.

In a previous study (Costa \& Figueiredo, under review) we have identified three groups of infants with three different psychophysiological profiles "withdrawn", "extroverted" and "underaroused". The identification of these psychophysiological profiles was determined according to the infants' neurobehavioral performance, social withdrawal and neuroendocrine reactivity to inoculation. (1) "Withdrawn" infants showed severe signs of social withdrawal, poor neurobehavioral performance as well as high neuroendocrine reactivity to a stressful stimulus; (2) "extroverted" infants showed practically no signs of social withdrawal, had a good neurobehavioral performance and a neuroendocrine reactivity to a stressful stimulus on the 
average, and the (3) "underaroused" infants showed some signs of social withdrawal, average neurobehavioral performance and a low neuroendocrine reactivity to a stressful stimulus.

In this study we intend to analyze (1) differences in infant temperament at 3 and 12 months according to infants' psychophysiological profiles: "withdrawn", "extroverted", and "underaroused" and (2) changes in infant temperament from 3 to 12 months, namely according to the infant psychophysiological profile and the quality of mother-infant interaction. We hypothesize that "extroverted" infants will be perceived as having a easier temperament compared to "withdrawn" or "underaroused" infants. Additionally, according to contemporaneous theories of temperament, we expect some changes although not in all dimensions of infant temperament from 3 to 12 months and specifically that these changes are dependent of both infant and characteristics and the quality of mother-interaction.

\section{Method}

\subsection{Sample}

The sample was composed of 94 infants. Most infants were born after a normal and full-term gestation. More than half were born through a distocic delivery and generally had no need for reanimation. At birth, the infants' height ranged from $45.90 \mathrm{~cm}$ to $54.00 \mathrm{~cm}(M=49.44 \mathrm{~cm}, S D=1.84)$, the cephalic perimeter ranged from $31 \mathrm{~cm}$ to $37 \mathrm{~cm}(M=34.60 \mathrm{~cm}, S D=1.29)$, weight ranged from $2450 \mathrm{~g}$ to $4055 \mathrm{~g}(M=3243 \mathrm{~g}, S D=424)$ and the ponderal index ranged from 2.24 to $3.29(M=2.71$, $S D=0.23)$. The apgar scores ranged from 5 to $10(M=8.63, S D=0.91)$ in the 1 st minute of life and from 8 to $10(M=9.76$, $S D=0.53$ ) in the 5 th minute of life (see Table 1 ).

\subsection{Procedures}

This research was conducted in two Health Care Centers $(N=94)$ in northern Portugal. In one Health Care center pregnant women were contacted during the second trimester of gestation, when attending the routine medical visits. $62 \%$ of the contacted women agreed to participate, 30\% declined participation alleging lack of time and $8 \%$ alleging lack of interest. In the other Health Care Center, mothers were contacted when attending the routine inoculation of their one-month-old infants. 96\% of the contacted mothers agreed to participate, $3 \%$ declined participation alleging lack of time and $1 \%$ alleging lack of interest. These Health Care Center have identical functioning and follow the guidelines of the Health Ministry. The discrepancy of research attendance may be due to differences on timing of recruitment. The exclusion criteria were incapability to read or write Portuguese and multiple gestations. The study's aims and procedures were explained, and the mothers signed an informed consent. All evaluation procedures were performed and videotaped either at the mothers' homes or at the Health Care Centers.

A socio-demographic questionnaire on the infants' medical data was filled out at birth and at 8 weeks of life ( \pm 5 days) the assessment using the Neonatal Behavioral Assessment Scale (NBAS, Brazelton \& Nugent, 1995) was performed and video-taped. This examination was conducted in a particular sequence by trained and reliable examiners halfway between feedings in a quiet and semi-darkened room with a temperature of $22-27^{\circ} \mathrm{C}$. The NBAS scores were taken immediately after performing the assessment and were later visualized with two purposes: (1) to analyze potential doubts on NBAS scoring and (2) to score the infant's performance on the Alarm Distress Baby Scale (ADBB, Guedeney \& Fermanian, 2001). At between 8 and 12 weeks of life, a saliva sample was collected from the infants' mouth before ( $5 \mathrm{~m})$ and after ( $20 \mathrm{~m})$ a

Table 1

Socio-demographic and medical data.

\begin{tabular}{|c|c|c|c|c|c|}
\hline Maternal and gestational data & & $(\%)$ & Neonatal data & & $(\%)$ \\
\hline Maternal age & $\begin{array}{l}\geq 20 \Lambda \leq 34 \\
>35\end{array}$ & $\begin{array}{r}96.0 \\
4.0\end{array}$ & Time of gestation & $\begin{array}{l}<37 \\
\geq 37 \Lambda \leq 40 \\
>40\end{array}$ & $\begin{array}{r}7.2 \\
82.1 \\
10.7\end{array}$ \\
\hline Year of education & $\begin{array}{l}<9 \\
\geq 9\end{array}$ & $\begin{array}{l}23.0 \\
77.0\end{array}$ & Gender & $\begin{array}{l}\text { Female } \\
\text { Male }\end{array}$ & $\begin{array}{l}46.9 \\
53.1\end{array}$ \\
\hline Marital status & $\begin{array}{l}\text { Married } \\
\text { Cohabiting }\end{array}$ & $\begin{array}{l}81.0 \\
19.0\end{array}$ & Reanimation at birth & $\begin{array}{l}\text { No } \\
\text { Yes }\end{array}$ & $\begin{array}{r}94.6 \\
5.4\end{array}$ \\
\hline Parity & $\begin{array}{l}\text { Primiparous } \\
\text { Multiple gest. }\end{array}$ & $\begin{array}{l}84.2 \\
15.8\end{array}$ & Weight & $\begin{array}{l}<2500 \mathrm{~g} \\
\geq 2500 \mathrm{~g}\end{array}$ & $\begin{array}{r}1.7 \\
98.3\end{array}$ \\
\hline Type of gestation & $\begin{array}{l}\text { Normal } \\
\text { Risk }\end{array}$ & $\begin{array}{l}80.4 \\
19.6\end{array}$ & Ponderal index & $\begin{array}{l}<2.5 \\
\geq 2.5\end{array}$ & $\begin{array}{l}13.5 \\
86.5\end{array}$ \\
\hline Type of delivery & $\begin{array}{l}\text { Eutocic } \\
\text { Distocic }\end{array}$ & $\begin{array}{l}34.2 \\
65.8\end{array}$ & Apgar index: 1 st m & $\begin{array}{l}<7 \\
\geq 7\end{array}$ & $\begin{array}{r}3.8 \\
96.2\end{array}$ \\
\hline Type of anaesthesia & $\begin{array}{l}\text { None } \\
\text { Epidural } \\
\text { General }\end{array}$ & $\begin{array}{r}2.6 \\
86.8 \\
10.5\end{array}$ & Type of feeding & $\begin{array}{l}\text { Beast-feed } \\
\text { Bottle-feed }\end{array}$ & $\begin{array}{l}89.4 \\
10.6\end{array}$ \\
\hline
\end{tabular}


routine inoculation. Between 12 and 16 weeks, the Infant Behavior Questionnaire (IBQ, Rothbart, 1981) was filled out by the mothers. The timing for the use of each evaluation instrument was according to specific instructions of the authors.

\subsection{Measures}

\subsubsection{Neonatal behavior}

The Neonatal Behavioral Assessment Scale (NBAS, Brazelton \& Nugent, 1995) assesses the newborn's competences across different developmental areas - autonomic, motor, states and social - and describes how these areas are integrated. The scale, composed of 28 behavioral and 18 reflex items, is suitable for examining newborns and infants up to 2 months old. By the end of the assessment, the examiner evaluates the neurobehavioral performance of the infant, describing his/her strengths, adaptive responses and possible vulnerabilities. The 28 items of the NBAS are scored on a 9-point scale and then subjected to clustering techniques which yield summary scores for habituation (response decrement to light, rattle, bell and foot stimulation), orientation (capacity to focus on and follow only a face, face and voice, ball, rattle, turn to a human voice or to the sound of a rattle and alertness), motor performance (evaluating the infants' general tone, motor maturity, pull-to-sit, defensive response and lability of skin color), range of state (peak of excitement, rapidity of build-up, irritability, lability of states), regulation of state (infants' cuddliness, consolability, self-quieting and hand-to-mouth), and autonomic stability (tremulousness, startles and activity level). Additional summary score indexes for abnormal reflexes were considered (number of abnormal reflexes through the evaluation-plantar grasp, babinski, ankle clonus, rooting, sucking, glabella, passive resistence of legs, passive resistence of arms, palmar grasp, placing, standing, walking, crawling, incurvation, tonic deviation of head/eyes, nystagmus, tonic neck reflex, moro). For the NBAS total score behavioral and reflexes items were recoded so that a better performance corresponded to higher score and were then added. The $\alpha$ of Cronbach of the scales ranged from .54 (autonomic stability) to .74 (range of state) (Costa et al., 2010), similar to those found in other studies (Moragas, Deu, Mussons, Boatella-Costa, \& Zurita, 2007).

\subsubsection{Social withdrawal}

The Alarm Distress Baby Scale (ADBB, Guedeney \& Fermanian, 2001) consists of eight items and aims to assess prolonged reactions of social withdrawal in infants. The ADBB was created to help assessing social withdrawal in children aged between 2 and 24 months, in the context of a pediatrician routine physical examination or a psychological assessment. To enable the observation of a child's behavioral response, the clinician must engage the infant in social behavior by talking, touching and smiling to him. The eight items, each one rated from zero to four (the lowest scores representing optimal social behavior), are facial expression; eye contact; general level of activity; self-stimulation gestures; vocalizations; briskness of response to stimulation; relationship with the observer, and attractiveness to the observer. The ADBB total score is given by the sum of the eight items. The higher the ADBB results the greater the signs of social withdrawal shown by the infant. The cut-off point of 5 resulted in the best sensitivity $(0.82)$ and specificity $(0.78)$ to detect infants at-risk (Guedeney \& Fermanian, 2001). The Portuguese version of the scale has a reasonable internal consistency ( $\alpha$ of Cronbach $=.60$ ) (Figueiredo \& Costa, 2008). The inter-rater reliability for this sample was calculated using an intra-class coefficient $($ ICC $=.92$ ).

\subsubsection{Neuroendocrine reactivity to inoculation}

Saliva samples were collected before $(5 \mathrm{~min})$ and after $(20 \mathrm{~min})$ routine inoculation between 8 and 12 weeks old. A cotton roll was placed in the infant's mouth for about 2-3 min and then put into a plastic tube (Salivette). On the day of the testing, all specimens were taken to the laboratory where they were centrifuged to remove mucus and then stored in a freezer $\left(-20^{\circ} \mathrm{C}\right)$. All saliva samples were assayed for cortisol concentration using a quimioluminescence method. Cortisol units are expressed in $\mu \mathrm{g} / \mathrm{dL}$. The difference between the post-test and pre-test cortisol was calculated and referred to as " $\delta$ cortisol".

\subsubsection{Infant temperament}

The Infant Behavior Questionnaire - Revised (IBQ-R, Gartstein \& Rothbart, 2003) is a revised version of the IBQ (Rothbart, 1981) which was designed to assess temperament in infants aged between 3 and 12 months. The Portuguese version used in this study was obtained after translation and back-translation of the aforementioned one. It is composed of 191 items scored on a 7 points Likert scale. It assesses the following 14 dimensions of temperament: (1) activity level: movement of arms and legs, squirming and locomotor activity. (2) Distress to limitations: baby's fussing, crying or showing distress while (a) in a confining place or position; (b) involved in caretaking activities; (c) unable to perform a desired action. (3) Approach: rapid approach, excitement, and positive anticipation of pleasing activities. (4) Fear: the baby's startle or distress to sudden changes in stimulation, novel physical objects or social stimuli; inhibited approach to novelty. (5) Duration of orienting: the baby's attention to and/or interaction with a single object for extended periods of time. (6) Smiling and laughter: the child's smile or laughter in general caretaking and playing situations. (7) Vocal reactivity: amount of vocalization exhibited by the baby in daily activities. (8) Sadness: general low mood; lowered mood and activity specifically related to personal suffering, physical state, object loss, or inability to perform a desired action. (9) Perceptual sensitivity: amount of detection of slight, low intensity stimuli from the external environment. (10) High intensity pleasure: amount of pleasure or enjoyment related to high stimulus intensity, rate, complexity, novelty, and incongruity. (11) Low intensity pleasure: amount of pleasure or enjoyment related to situations involving low stimulus intensity, rate, complexity, novelty, and incongruity. (12) Cuddliness: the baby's expression of enjoyment and shaping of the body to express desire of being held by a caregiver. (13) Soothability: baby's 
reduction of fussing, crying, or distress when the caretaker uses soothing techniques. (14) Falling reactivity/rate of recovery from distress: rate of recovery from peak distress, excitement, or general arousal; ease to fall asleep. Three dimensions of temperament were computed: (1) surgency/extraversion-computed from the mean scores of approach, vocal reactivity, high intensity pleasure, smiling and laughter, activity level and perceptual sensitivity; (2) negative affectivity-computed from the mean scores of distress to limitation, fear and falling reactivity; (3) orienting/regulation-computed from the mean scores of low intensity pleasure, cuddliness, duration of orienting and soothability.

The IBQ reliability is good (Rothbart, 1986). Cronbach's alpha for surgency/extraversion was 0.92, with estimates for the negative affectivity and orienting/regulation factors both equaling 0.91 (Gartstein \& Rothbart, 2003). The Portuguese version of this instrument shows good internal consistency in all the sub-scale with $\alpha$ of Cronbach ranging from 0.70 to 0.93 (Costa \& Figueiredo, submitted for publication).

\subsubsection{Mother-infant interaction}

The Global Rating Scales (GRS, Gunning, Fiori-Cowley, \& Murray, 1999; Murray, Fiori-Cowley, Hooper, \& Cooper, 1996) were developed to assess in the quality of mother-infant interaction. This is a video-based assessment of the quality of the mother-infant engagement that can be applied from 2 to 6 months post-partum. Mothers were instructed simply to play with their infants in any way they choose without the use of toys. The mother was asked to sit in front of the infant and play with him/her for a 5 min face-to-face playing session. A video camera was set up to film the event in order to obtain a full image of the infants' body, and the mother's full-face image was also filmed using a mirror placed adjacently to the infant. During a 5 min video-recorded assessment of free play between mother and infant, the scales globally assess the quality of: (1) maternal behavior, (2) infant behavior, and (3) overall interaction between the two.

In this study we only used the quality of the overall interaction between mother and infant: (1) good-poor composed of the average score of 5 items (smooth/easy vs. difficult, fun vs. serious, satisfying vs. unsatisfying, much engagement vs. no engagement, excited engagement vs. quiet engagement), a sum score of 5 is "good interaction" and near 1 "poor interaction". This scale rates the nature of the engagement between mother and infant. The GRS scales are a reliable instrument for the assessment of mother-infant interaction (Murray et al., 1996). They are valid cross-culturally for women with or without psychopathology (Gunning et al., 2004). In this study, a researcher trained by the Winnicott Research Unit carried out the procedure and scored the mother-infant interactions. The researcher achieved the required reliability (at least $90 \%$ of the scores were within one point of the original score and $45 \%$ were exactly the same).

\subsection{Data reduction and statistical analyses}

Several multivariate analyses of variance (MANOVAs) were performed to identify potential differences in maternal perception of infant temperament at ages of 3 and 12 months in three groups of infants with three different psychophysiological profiles "Withdrawn", "Extroverted" and "Underaroused". The identification of these psychophysiological profiles was performed through cluster analysis - according to the infants' neurobehavioral performance, social withdrawal and neuroendocrine reactivity to inoculation - and is described elsewhere (Costa \& Figueiredo, under review).

Mixed-model ANOVAs with between-subjects factors were performed to identify changes in the maternal perception of infant temperament at ages from 3 to 12 months old and the potential effect of the infants' psychophysiological profile on those changes. The interest was to understand the differential effect of different temperament dimensions, and so the temperament dimensions and not factors were used in this analysis. The model considers temperament dimensions as measures and time moments as within-subjects factors. The infant's psychophysiological profile was considered as betweensubjects factor. Sphericity assumption is not an issue in this analysis, seen that there are only two levels of within-subjects factors (Field, 2005).

Mixed-model ANOVAs with between subject factors were performed to identify changes on maternal perception of infant temperament from 3 to 12 months old and the potential effect of the quality of mother-infant interaction on those changes. For this analysis, two groups were composed based on the mean score of the GRS. Those below the sample mean were considered "poor" interaction while those above the sample mean were considered "good" interaction. The model included the quality of mother-infant interaction as measures and time moments as within-subjects effects factor. Mother-infant interaction was considered as between-subjects factors. Sphericity assumption is not an issue in this analysis once there are only two levels of the within-subjects factor (Field, 2005).

\section{Results}

\subsection{Infant psychophysiological profile and temperament at 3 months}

Several multivariate analyses of variance (MANOVAs) were performed to identify potential differences in the maternal perception of infant temperament at the age of 3 months in three groups of infants with different psychophysiological profiles "withdrawn", "extroverted" and "underaroused". The MANOVA is not significant $(\Lambda=1.414 ; F(2,96)=1.414 ; p=.263)$. Subsequent univariate analyses followed by the Bonferroni post hoc test, indicated a significant effect for infant distress, cuddliness, approach and vocal reactivity (see Table 2). Compared to extroverted infants, withdrawn infants have a higher score on distress to limitation (IC95\%].40, 1.38[; $p=.038$ ) and underaroused infants have higher scores on approach (IC95\%]-2.68, 
Table 2

Univariate F-test: differences between groups on mothers' perception of infant temperament at 3 months old.

\begin{tabular}{|c|c|c|c|c|c|c|}
\hline & $\begin{array}{l}\text { Withdrawn }(A) \\
(n=16) \\
M(S D)\end{array}$ & $\begin{array}{l}\text { Extroverted (B) } \\
(n=56) \\
M(S D)\end{array}$ & $\begin{array}{l}\text { Underaroused }(C) \\
(n=25) \\
M(S D)\end{array}$ & $F$ & $p$ & \\
\hline Distress & $4.38(.39)$ & $3.89(.83)$ & $4.31(.62)$ & 3.482 & .030 & A vs. B \\
\hline Cuddliness & $5.81(.83)$ & $6.14(.58)$ & $6.37(.57)$ & 3.224 & .034 & A vs. C \\
\hline Approach & $4.81(1.22)$ & $5.85(1.39)$ & $6.64(1.35)$ & 5.210 & .011 & B vs. C \\
\hline Vocal reactivity & $4.42(.99)$ & $4.83(1.24)$ & $5.28(.79)$ & 3.284 & .044 & A vs. C \\
\hline
\end{tabular}

Table 3

Univariate F-test: differences between groups on mothers' perception of infant temperament at 12 months old.

\begin{tabular}{|c|c|c|c|c|c|c|}
\hline & $\begin{array}{l}\text { Withdrawn }(\mathrm{A}) \\
(n=16) \\
M(S D)\end{array}$ & $\begin{array}{l}\text { Extroverted (B) } \\
(n=56) \\
M(S D)\end{array}$ & $\begin{array}{l}\text { Underaroused }(\mathrm{C}) \\
(n=25) \\
M(S D)\end{array}$ & $F$ & $p$ & \\
\hline Activity level & $3.09(1.11)$ & $4.54(.95)$ & $4.08(.86)$ & 4.864 & .022 & A vs. B \\
\hline Smiling/laughter & $4.90(.44)$ & $5.73(.62)$ & $5.60(.66)$ & 3.278 & .058 & A vs. B \\
\hline Perceptual sensitivity & $5.46(.98)$ & $4.47(.67)$ & $4.08(1.47)$ & 3.946 & .030 & A vs. C \\
\hline
\end{tabular}

$-.26[; p=.010$ ). Compared to underaroused infants, withdrawn infants have lower scores on cuddliness (IC95\%]-1.41, -.29[; $p=.040)$ and vocal reactivity (IC95\%] $-2.28,-.55[; p=.041)$.

\subsection{Infant psychophysiological profile at 3 months and temperament at 12 months}

A multivariate analysis of variance (MANOVA) was performed to identify potential group differences on mothers' perceptions of infant temperament at 12 months. The MANOVA is marginally significant $(\Lambda=.044 ; F(2,96)=2.142 ; p=.056)$. Subsequent univariate analysis followed by the Bonferroni post hoc test, indicated a significant effect for infant activity level and perceptual sensitivity and a marginally significant effect for smiling/laughter (see Table 3). Compared to extroverted infants, withdrawn infants have lower scores on activity level (IC95\%]-3.23, -.31 [; $p=.020$ ) and on smiling/laughter (IC95\%]-1.71, $-.05[; p=.060)$. Compared to underaroused infants, withdrawn infants have higher scores on perceptual sensitivity (IC95\%].74, 3.50[; $p=.034)$.

\subsection{Differences on infant's temperament from 3 to 12 months}

Mixed-model ANOVAs were performed in order to analyze differences on maternal perception of infant temperament from 3 to 12 months old. The homogeneity of variances/covariances was tested with the $M$ Box test $(M=63.270 ; F=1.421$; $p=0.101)$ and it is valid. The within-subjects analyses reveal that marginally significant differences in the maternal perception of infant temperament occur between 3 and 12 months regarding the maternal perception of infant's distress to limitation $(F=4.200, p=.057)$ and approach $(F=4.336, p=.054)$, and significant differences regarding the infant's cuddliness $(F=5.691$, $p=.030)$ and sadness $(F=12.735, p=.003)$. Older infants have lower scores on distress to limitation, cuddliness and sadness and higher scores on approach (see Table 4).

Table 4

Mixed-model ANOVA: changes on mothers' perception of infant temperament from 3 to 12 months old.

\begin{tabular}{|c|c|c|c|c|}
\hline & $\begin{array}{l}3 \text { months } \\
M(S D)\end{array}$ & $\begin{array}{l}12 \text { months } \\
M(S D)\end{array}$ & $F$ & $p$ \\
\hline Activity level & $4.48(1.70)$ & $4.20(0.99)$ & .051 & .824 \\
\hline Distress & $4.52(0.93)$ & $3.85(0.77)$ & 4.200 & .057 \\
\hline Fear & $4.14(2.05)$ & $3.17(1.40)$ & .519 & .482 \\
\hline Duration/orientation & $5.30(1.98)$ & $4.19(1.17)$ & .800 & .384 \\
\hline Smilling/laughter & $5.24(1.69)$ & $5.64(0.64)$ & 3.583 & .077 \\
\hline High pleasure & $5.68(1.45)$ & $5.80(0.66)$ & 1.536 & .233 \\
\hline Low pleasure & $5.40(1.50)$ & $5.44(0.89)$ & .584 & .456 \\
\hline Soothability & $5.89(1.09)$ & $5.22(0.73)$ & 1.668 & .215 \\
\hline Falling reactivity & $5.14(1.10)$ & $5.11(1.01)$ & .689 & .419 \\
\hline Cuddliness & $6.25(0.92)$ & $5.43(0.70)$ & 5.691 & .030 \\
\hline Perceptual sensitivity & $5.37(1.66)$ & $4.72(1.30)$ & 1.098 & .310 \\
\hline Sadness & $4.88(1.40)$ & $3.44(0.84)$ & 12.735 & .003 \\
\hline Approach & $5.15(1.28)$ & $5.70(0.71)$ & 4.336 & .054 \\
\hline Vocal reactivity & $5.50(1.37)$ & $5.35(0.77)$ & .019 & .892 \\
\hline
\end{tabular}


Table 5

Mixed-model ANOVA: changes on mothers' perception of infant temperament from 3 to 12 months old according to infant psychophysiological profile.

\begin{tabular}{|c|c|c|c|c|c|c|c|c|}
\hline & \multicolumn{2}{|l|}{ Withdrawn } & \multicolumn{2}{|l|}{ Extroverted } & \multicolumn{2}{|c|}{ Underaroused } & \multirow[t]{2}{*}{$F$} & \multirow[t]{2}{*}{$p$} \\
\hline & $\begin{array}{l}3 \text { months } \\
M(S D)\end{array}$ & $\begin{array}{l}12 \text { months } \\
M(S D)\end{array}$ & $\begin{array}{l}3 \text { months } \\
M(S D)\end{array}$ & $\begin{array}{l}12 \text { months } \\
M(S D)\end{array}$ & $\begin{array}{l}3 \text { months } \\
M(S D)\end{array}$ & $\begin{array}{l}12 \text { months } \\
M(S D)\end{array}$ & & \\
\hline Fear & $4.38(1.31)$ & $4.00(1.00)$ & $3.21(1.81)$ & $3.75(1.18)$ & $2.88(1.39)$ & $3.85(.96)$ & 3.648 & .045 \\
\hline Smilling/laughter & $5.05(1.39)$ & $4.90(.44)$ & $5.22(1.21)$ & $5.73(.62)$ & $5.16(1.46)$ & $5.60(.66)$ & 4.448 & .029 \\
\hline Approach & $4.81(.22)$ & $4.78(1.11)$ & $5.85(1.39)$ & $5.92(1.26)$ & $6.64(1.35)$ & $6.71(1.18)$ & 3.856 & .042 \\
\hline
\end{tabular}

Table 6

Mixed-model ANOVA: changes on mothers' perception of infant temperament from 3 to 12 months old according to quality of mother-infant interaction.

\begin{tabular}{|c|c|c|c|c|c|c|}
\hline & \multicolumn{2}{|c|}{ Poor interaction } & \multicolumn{2}{|c|}{ Good interaction } & \multirow[t]{2}{*}{$F$} & \multirow[t]{2}{*}{$p$} \\
\hline & $\begin{array}{l}3 \text { months } \\
M(S D)\end{array}$ & $\begin{array}{l}12 \text { months } \\
M(S D)\end{array}$ & $\begin{array}{l}3 \text { months } \\
M(S D)\end{array}$ & $\begin{array}{l}12 \text { months } \\
M(S D)\end{array}$ & & \\
\hline Activity level & $4.30(1.50)$ & $4.40(.86)$ & $4.06(1.45)$ & $4.00(.80)$ & 3.466 & .047 \\
\hline Smilling/laughter & $5.34(1.44)$ & $5.09(0.70)$ & $5.47(1.38)$ & $5.97(0.74)$ & 4.638 & .024 \\
\hline High pleasure & $5.64(1.37)$ & $5.45(0.57)$ & $5.93(1.31)$ & $5.98(0.64)$ & 3.928 & .043 \\
\hline
\end{tabular}

\subsection{Infant psychophysiological profile and changes on temperament from 3 to 12 months}

To analyze the potential effect of the infant psychophysiological profile on changes of mothers' perception of infant temperament, the infants' psychophysiological profile was considered as between-subjects factors in the mixed-model ANOVA. The results show that the infant psychophysiological profile has a significant effect on the pattern of change of mothers' perception of some dimensions of infant temperament, including fear $(\Lambda=.846, F=3.648, p=.045)$, $\operatorname{smiling}(\Lambda=.792, F=4.448$, $p=.029)$ and approach $(\Lambda=.923, F=3.856, p=.042)$. Mothers of withdrawn infants perceived their infants' expressions of fear decreasing over time, while mothers of extroverted (IC95\%]-1.38, $-.55[; p=.032$ ) and underaroused (IC95\%]-2.04, $-1.20[; p=.044)$ infants perceived their infants' expressions of fear to increase over time. Mothers of withdrawn infants perceived their infants' to smile less over time, while mothers of extroverted (IC95\%]-2.48, $-.13[; p=.028$ ) and underaroused (IC95\%]-1.89,-.30[; $p=.046$ ) infants perceived their infants' to smile more over time. Mothers of withdrawn infants perceived their infants' approach to decrease over time, while mothers of extroverted (IC95\%]-1.23, $-.64[; p=.037)$ and underaroused (IC95\%]-1.04, $-.51[; p=.048$ ) infants perceived their infants' approach to increase over time (see Table 5).

\subsection{Mother-infant interaction and changes in infant temperament from 3 to 12 months}

To analyze the potential effect of the quality of mother-infant interaction (good vs. poor) on changes on mothers' perception of infant temperament, the quality of mother-infant interaction (good vs. poor) was considered as between-subjects factors in the mixed-model ANOVA. The results show that the quality of mother-infant interaction has a significant effect on the pattern of changes on mothers' perception of some dimensions of infant temperament, including high pleasure $(\Lambda=.745$, $F=3.928, p=.043)$, smiling $(\Lambda=.692, F=4.638, p=.024)$ and activity level $(\Lambda=.843, F=3.466, p=.047)$. A poor mother-infant interaction is associated with decreases in mothers' perception of infant high pleasure (IC95\%]1.54, 2.05[; $p=.042$ ), in mothers' perception of infants' smile (IC95\%]-2.04, -.56[; $p=.038$ ) and increases in mothers' perceptions of infant level of activity (IC95\%]-1.56, .-54[; $p=.043$ ) (see Table 6).

\section{Discussion}

This study was design to understand differences in infant temperament at 3 and 12 months according to infants' psychophysiological profiles: "withdrawn", "extroverted”, and "underaroused", changes in infant temperament from 3 to 12 months namely according to the infant psychophysiological profile and the quality of mother-infant interaction. We hypothesize that "extroverted" infants would be perceived as having a easier temperament compared to "withdrawn" or "underaroused" infants. Additionally we expected changes in some infant temperament dimensions from 3 to 12 months and specifically that these changes would be dependent of both infant characteristics and the quality of mother-interaction.

As hypothesized, mothers' perception of infants' temperament depends on the infant psychophysiological profile. This means that infants' hormonal, social and emotional behaviors displayed early in life, before the development of diverse social interactions, may moderate infant temperament. Mothers' perception of extroverted infants differs from maternal perception of withdrawn infants in that the first ones show more approach, excitement and positive anticipation of pleasurable activities. Mothers' of withdrawn infants perceived them as having less cuddliness behaviors and vocal reactivity than mothers of underaroused infants. Withdrawn infants' temperament seems to fit Thomas et al. (1968) "difficult" type of temperament. "Difficult" children have irregular behavior, intense reactions, are withdrawn from stimulus, have difficulty to adapt and negative mood. Accordingly, withdrawn infants have difficulties in engaging in new activities, approaching and giving vocal 
feedback compared to other infants. At 12 months, withdrawn infants are perceived by their mothers as having lower levels of activity and smiling/laughter compared to extroverted infants as well as higher perceptual sensitivity compared to underaroused infants. At both 3 and 12 months withdrawn infants seem to be perceived by their mothers as more difficult, which might place them in a more vulnerable position for later personality and social development difficulties (Kagan, 1998; Rothbart \& Bates, 1998). According to Thomas et al. (1968), behavioral disorder appears when there is a conflict between a child's temperament and his environmental characteristics, and given the difficulty of withdrawn infants' temperament, there is a higher probability that this conflict might occur.

Additional evidence was gathered regarding our second hypothesis, since stability was found in mothers' perception of infant temperament from 3 to 12 months on most (although not all) temperament dimensions: activity level, fear, duration/orientation, smiling/laughter, high pleasure, low pleasure, soothability, falling reactivity, perceptual sensitivity and vocal reactivity. Worobey and Blajda (1989) also found that the activity level, responsivity, and irritability remained stable from 2 to 12 months. Other studies found stability in temperament dimensions reflecting difficultness (Belsky, Rovine, \& Fish, 1989; Lee \& Bates, 1985) or emotionality (Matheny et al., 1985; Riese, 1987).

Nonetheless changes were noticed in distress to limitation, approach, cuddliness and sadness. Older infants have lower scores in distress to limitation, cuddliness and sadness and higher scores in approach. As occurred in our study, other authors also pointed out that there was an increment of approach in the first year of life (Rothbart, 1981). But, in discrepancy to our results, others have reported increases in activity level, distress to limitation and fear (Carranza et al., 2000; Rothbart, 1986, $1988,1989)$. Some authors argue that the original characteristics of temperament tend to remain constant in quality over the years, although in the course of development, the environmental circumstances may influence the infants' reactions and behavior (Thomas et al., 1968). Our results give empirical support to this premise of some stability in the expression of infant temperament, since ten dimensions of infant temperament remain stable over the first year of life (Rothbart \& Derryberry, 1981).

This study also provides evidence of the influence of infants' psychophysiological profile on changes on mothers' perception of infant temperament over the 1 st year of life as predicted, including fear, smiling and approach. This brings out the fact that the complex social emotional behaviors, displayed early in life, have an influence on later changes in the mother's perceptions of infant temperament. This result adds importance to the role of infant factors, which have been downplayed in previous studies that have only emphasized the influence of maternal and family factors, such as emotional cohesiveness of the family, maternal expressiveness (Matheny, 1986) and sensitivity (Washington et al., 1986) and marital problems (Engfer, 1986) on infant temperament. Mothers of withdraw infants do not perceive their expressions of fear to increase over the first year of life, this might be due to the fact that these infants withdraw from stimulus more than other infants and as such are not as subject as others to take risks. Since fear is an important dimension associated with the infant attachment style (Kochanska, 1998), this result should be further explored in future research.

Additionally, as hypothesized the quality of mother-infant interaction also influences changes in mothers' perception of infant temperament. Good patterns of mother-infant interaction are related to increases in the infants' positive emotionality and activity level. These results corroborate those of Belsky, Fish, and Isabella (1991) who have also found that the quality of mother-infant interaction is an important factor accounting for changes in infant temperament from 3 to 9 months. It seems that poor interactive experiences, with insensitive and unresponsive mothers may originate distress in the quality of care, leading the infant to express less positive emotionality (Belsky et al., 1991). Additionally, the infant may present more trouble developing competences to deal more effectively with negative emotionality (Belsky et al., 1991).

Conversely, a higher quality of maternal interaction seems to improve the infant's capacity of self-regulation and lead to positive changes in negative emotionality (Belsky et al., 1991). These results corroborate the theories that sustain that the environment might influence changes in infant temperament over time (Rothbart \& Derryberry, 1981).

This study adds to previous knowledge that the infant' social and emotional behaviors displayed early in life, before the development of diverse social interactions, can be differentiated by their mothers. Furthermore, mothers of withdrawn infants perceive them as more difficult. Considering the fact that our first study indicated relational difficulties in dyads of withdrawn infants, we might speculate that those difficulties may also be due to the infant temperament particularities. The fact that this group of infants are seen by their mothers as more difficult and that there are difficulties in the relational level may be indicative of an increased probability of developmental problems. Parental perceptions of infant behavior shape their own behavior and interaction with the infant (Crockenberg \& McCluskey, 1986). Considering the importance of parental-infant interaction for infant development, how parents (mis)interpret infant's signs may be a relevant issue for understanding child development outcomes. In fact, data suggests that the quality of interaction may influence the development of these temperamental difficulties: it seems that when a good interaction is established an increased positive emotionality is possible to develop. In turn this may have a potential positive impact on the mother-infant interaction and act as a protective factor to the infant development.

This study's major contribution relies on providing evidence regarding individual differences early in life, considering the infant's psychophysiological characteristics, and its impact on his/her mother's perception of infant temperament. Additionally, this study contributes to the knowledge of the environmental factors that influence the development of those individual differences. Limitation in this study include the sample size, the use of only two time points that limit our ability to assess stability and change in temperament and the use of only one independent temperament rater does not allow us to distinguish rater effects from infant effects. Further research should comprise a larger sample and study the developmental pathways of these different groups of infants, namely in terms of their personality and child development. 


\section{Acknowledgements}

We would like to thank the participants in this study and the Primary Health Center of Espinho and Santa Maria da Feira. We gratefully acknowledge the work of Filomena Louro of the Scientific Editing Programme of University of Minho for revising this article. This research was funded under an FCT PhD Grant (SFRH/BD/18249/2004).

\section{References}

Bates, J. E. (1987). Temperament in infancy. In J. D. Osofsky (Ed.), Handbook of infant development (2nd ed., pp. 1101-1149). New York: John Wiley \& Sons, Inc.

Belsky, J., Fish, M., \& Isabella, R. (1991). Continuity and discontinuity in infant negative and positive emotionality: Family antecedents and attachment consequences. Developmental Psychology, 27, 421-431.

Belsky, J., Hsieh, K., \& Crnic, K. (1998). Mothering, fathering and infant negativity as antecedents of boys' externalizing problems and inhibition at age 3: Differential susceptibility to rearing influence? Development and Psychopathology, 10, 301-319.

Belsky, J., Rovine, M., \& Fish, M. (1989). The developing family system. In M. Gunnar, \& E. Thelen (Eds.), Minnesota symposia on child psychology: Vol. 22. Systems and development (pp. 119-166). Hillsdale, NJ: Lawrence Erlbaum Associates.

Biederman, J., Rosenbaum, J. F., Bolducmurphy, E. A., Faraone, S. V., Chaloff, J., Hirshfeld, D. R., et al. (1993). A 3-year follow-up of children with and without behavioral-inhibition. Journal of the American Academy of Child and Adolescent Psychiatry, 32(4), 814-821.

Brazelton, T. B., \& Nugent, J. K. (1995). Neonatal Behavioral Assessment Scale (3rd ed.). London: Mac Keith Press.

Buss, A. H., \& Plomin, R. (1984). Temperament: Early developing personality traits. Hillsdale, NJ: Erlbaum.

Campbell, D. W., \& Eaton, W. O. (1999). Sex differences in the activity level of infants. Infant and Child Development, 8, 1-17.

Carranza, J. A., Perez-Lopez, J., Gonzalez, C., \& Martinez-Fuentes, M. T. (2000). A longitudinal study of temperament in infancy: Stability and convergence of measures. European Journal of Personality, 14, 21-37.

Costa, R., Figueiredo, B., Tendais, I., Conde, A., Pacheco, A., \& Teixeira, C. (2010). Brazelton Neonatal Behavioral Assessment Scale: A psychometric study in a Portuguese sample. Infant Behavior and Development, 33(4), 510-517.

Costa, R., \& Figueiredo, B. Infants' psychophysiological profile and mother-infant interaction, under review.

Costa, R., \& Figueiredo, B. Infant behavior questionnaire-revised: A psychometric study in a Portuguese sample, submitted for publication.

Crockenberg, S. B., \& McCluskey, K. (1986). Change in maternal behavior during the baby's first year of life. Child Development, 57, 746-753.

Else-Quest, N. M., Hyde, J. S., Goldsmith, H. H., \& Van Hulle, C. A. (2006). Gender differences in temperament: A meta-analysis. Psychological Bulletin, 132(1), 33-72.

Engfer, A. (1986). Antecedents of perceived behavior problems in infancy. In G. A. Kohnstamm (Ed.), Temperament discussed (pp. 165-180). Berwyn, PA: Swets North America.

Feldman, R., Greenbaum, C. W., Mayes, L. C., \& Erlich, S. H. (1997). Change in mother-infant interactive behavior: Relations to change in the mother, the infant, and the social context. Infant Behavior and Development, 20(2), 151-163.

Field, A. (2005). Discovering statistics using SPSS. London: SAGE Publications.

Figueiredo, B., \& Costa, R. (2008). Estudo de validação da versão portuguesa da Alarm Distress Baby Scale (ADBB). Acta Pediátrica Portuguesa, 39(5), 183-189.

Gartstein, M. A., \& Bateman, A. E. (2008). Early manifestations of childhood depression: Influences of infant temperament and parental depressive symptoms. Infant and Child Development, 17, 223-248.

Gartstein, M. A., \& Rothbart, M. K. (2003). Studying infant temperament via the revised infant behavior questionnaire. Infant Behavior E' Development, 26, $64-86$.

Goldsmith, H. H., Buss, A., Plomin, R., Rothbart, M. K., Thomas, A., Chess, S., et al. (1987). Roundtable: What is temperament? Four approaches. Child Development, 58, 505-529.

Goldsmith, H. H., \& Campos, J. J. (1986). Fundamental issues in the study of early temperament: The Denver twin temperament study. In M. E. Lamb, A. L. Brown, \& B. Rogoff (Eds.), Advances in developmental psychology (pp. 231-283). Hillsdale, NJ: Erlbaum.

Guedeney, A., \& Fermanian, J. (2001). A validity and reliability study of assessment and screening for sustained withdrawal reaction in infancy: The alarm distress baby scale. Infant Mental Health Journal, 22(5), 559-575.

Gunning, M., Conroy, S., Valoriani, V., Figueiredo, B., Kammerer, M., Muzik, M., et al. (2004). Measurement of mother-infant interactions and the home environment in a European setting: preliminary results from a cross-cultural study. British Journal of Psychiatry, 184s, s38-s44.

Gunning, M., Fiori-Cowley, A., \& Murray, L. (1999). The global ratings of mother-infant interaction-scoring manual (2nd ed.). Reading: Winnicott Research Unit, University of Reading.

Kagan, J. (1998). Biology and the child. In W. Damon, \& N. Eisenberg (Eds.), Handbook of child psychology: Social, emotional and personality development. New York: Wiley.

Kagan, J., Snidman, N., Zentner, M., \& Peterson, E. (1999). Infant temperament and anxious symptoms in school age children. Development E' Psychopathology, 11(2), 209-224.

Kochanska, G. (1998). Mother-child relationship, child fearfulness, and emerging attachment: A short-term longitudinal study. Developmental Psychology, 34(3), 480-490.

Lee, C. L., \& Bates, J. E. (1985). Mother-child interaction at age two years and perceived difficult temperament. Child Development, 56(5), $1314-1325$.

Lindhout, I. E., Markus, M. T., Hoogendijk, T. H., \& Boer, F. (2009). Temperamet and parental child-rearing style: Unique contributions to clinical anxiety disorders in childhood. European Child and Adolescent Psychiatry, 18(7), 439-446.

Martin, R. P., Wisenbaker, J., Baker, J., \& Huttunen, M. O. (1997). Gender differences in temperament at six months and five years. Infant Behavior \& Development, 20, 339-347.

Matheny, A. P. (1986). Stability and change of infant temperament: Contributions from the infant, mother, and family environment. In G. Kohnstamm (Ed.), Temperament discussed (5th ed., Vol. 3, pp. 49-58). Berwyn, PA: Swets North America.

Matheny, A. P., Riese, M. L., \& Wilson, R. S. (1985). Rudiments of infant temperament: Newborn to nine months. Developmental Psychology, 21, 486-494.

Maziade, M., Boudreault, M., Thivierge, J., Caperaa, P., \& Cote, R. (1984). Infant temperament: SES and gender differences and reliability of measurement in a large Quebec sample. Merrill-Palmer Quarterly, 30, 213-226.

McNeil, T. F., \& Persson-Blennow, I. (1988). Stability of temperament characteristics in childhood. American Journal of Orthopsychiatry, 58(4), 622-626.

Moragas, C. C., Deu, A. F., Mussons, F. B., Boatella-Costa, E., \& Zurita, M. L. (2007). Evaluación psicométrica de la Escala de Brazelton en una muestra de recién nacidos espanoles. Psicothema, 19(1), 140-149.

Murray, L., Fiori-Cowley, A., Hooper, R., \& Cooper, P.J. (1996). The impact of postnatal depression and associated adversity on early mother-infant interactions and later infant outcome. Child Development, 67, 2512-2626.

Riese, M. L. (1987). Temperamental stability between the neonatal period and 24 months. Developmental Psychology, 23, $216-222$.

Rosenbaum, J. F., Biederman, J., Bolduc-Murphy, E. A., Faraone, S. V., Chaloff, J., Hirshfeld, D. R., et al. (1993). Behavioral inhibition in childhood: A risk factor for anxiety disorders. Harvard Review of Psychiatry, 1(1), 2-16.

Rothbart, M. K. (1981). Measurement of temperament in infancy. Child Development, 52, 569-578.

Rothbart, M. K. (1986). Longitudinal observation of infant temperament. Developmental Psychology, 22, 356-365.

Rothbart, M. K. (1988). Temperament and the development of the inhibited approach. Child Development, 59, 1241-1250. 
Rothbart, M. K. (1989). Temperament and development. In G. A. Kohnstamm, J. E. Bates, \& M. K. Rothbart (Eds.), Temperament in childhood (pp. 187-248). New York: Wiley.

Rothbart, M. K., \& Bates, J. E. (1998). Temperament. In W. (Series Ed. ) Damon, \& N. (Vol. Ed. ) Eisenberg (Eds.), Handbook of child psychology: Vol. 3. Social, emotional, and personality development (5th ed., pp. 105-176). New York: Wiley.

Rothbart, M. K., \& Derryberry, D. (1981). Development of individual differences in temperament. In M. E. Lamb, \& A. L. Brown (Eds.), Advances in developmental psychology (pp. 37-86). Hillsdale, NJ: Erlbaum.

Rothbart, M. K., Derryberry, D., \& Posner, M. I. (1994). A psychobiological approach to the development of temperament. In J. E. Bates, \& T. D. Wachs (Eds.), Temperament: Individual differences and the interface of biology and behavior (pp. 83-116). Washington, DC: American Psychological Association.

Rothbart, M. K., \& Posner, M. I. (1985). Temperament and the development of self-regulation. In L. C. Hartlage, \& C. F. Telzrow (Eds.), The neuropsychology of individual differences: A developmental perspective (pp. 93-123). New York: Plenum.

Ruff, H. A., \& Rothbart, M. K. (1996). Attention in early development: Themes and variations. New York: Oxford University Press.

Seifer, R., Schiller, M., Sameroff, A. J., Resnick, S., \& Riordan, K. (1996). Attachment, maternal sensitivity, and temperament during the first year of life. Developmental Psychology, 32, 3-11.

Thomas, A., Chess, S., \& Birch, H. G. (1968). Temperament and behavior disorders in children. New York: New York University Press.

Washington, J., Minde, K., \& Goldberg, S. (1986). Temperament in preterm infants: Style and stability. Journal of the American Academy of Child and Adolescent Psychiatry, 25, 493-502.

Wolke, D., Rizzo, P., \& Woods, S. (2002). Persistent infant crying and hyperactivity problems in middle childhood. Pediatrics, $109,1054-1060$.

Worobey, J., \& Blajda, V. M. (1989). Temperament ratings at 2 weeks, 2 months, and 1 year: Differential stability of activity and emotionality. Developmental Psychology, 25(2), 257-263. 